\title{
Laparoscopic total gastrectomy with distal pancreatosplenectomy and D2 lymphadenectomy for advanced gastric cancer
}

\author{
Ichiro Uyama, Atsushi Sugioka, Junko Fujita, Yoshiyuki Komori, Hideo Matsui, and Akitake Hasumi \\ Department of Surgery, Fujita Health University, School of Medicine, 1-98 Dengakugakubo, Kutsukake-cho, Toyoake, Aichi 470-1192, Japan
}

\begin{abstract}
:
The standard lymph node dissection for advanced gastric cancer is a D2 dissection. Although D2 laparoscopy-assisted total gastrectomy with distal pancreatosplenectomy has been reported, no studies have reported a completely intraabdominal laparoscopic approach, because of the technical difficulty of the procedure. We successfully performed this novel procedure in two patients with advanced gastric cancer located in the upper portion of the stomach. In fact, this surgery is technically feasible, and has a potential curability comparable with that of open surgery.
\end{abstract}

Key words: laparoscopic surgery, total gastrectomy, pancreatosplenectomy, lymph node dissection, gastric cancer

\section{Introduction}

In recent years, laparoscopic surgery has been used in the treatment of early gastric malignancies [1-6], although there are a few reports of laparoscopic radical gastrectomy for advanced gastric cancer [7,8]. Although the D2 laparoscopy-assisted total gastrectomy with distal pancreatosplenectomy was reported, by Azagra et al. [7], no studies have reported a completely laparoscopic approach.

Recently, we successfully performed completely intra-abdominal laparoscopic total gastrectomy with distal pancreatosplenectomy and D2 lymphadenectomy in two patients with advanced gastric cancer. We describe here our surgical procedure and the initial clinical results.

Offprint requests to: I. Uyama

Received on Aug. 31, 1999; accepted on Jan. 27, 2000

\section{Patients}

We performed this surgery in two men, aged 71 years and 50 years, respectively. In both of these patients, the gastroendoscopic findings were advanced gastric cancer (c type 2) located in the upper portion. According to the Japanese classification of gastric carcinoma (JCGC) by the Japanese Research Society for Gastric Cancer [9] and the Japanese Gastric Cancer Association [10], both patients' clinical findings were $\mathrm{cT} 2, \mathrm{cN} 0, \mathrm{cH} 0, \mathrm{cPO}$, and cM0, cStage IB.

We explained the operative procedure and the advantages and risks, and our experience with laparoscopic surgery, to the patients preoperatively. Both patients were notified of their cancer diagnoses preoperatively.

\section{Surgical technique}

The numbers and groups of regional lymph nodes were defined according to the JCGC.

General anesthesia was induced and the patient was placed in the reverse Trendelenburg position with the legs apart so that the transverse colon and small bowel fell toward the lower quadrant. The surgeon stood on the patient's right, with the first assistant on the patient's left, and the camera operator stood between the patient's legs.

After pneumoperitoneum was established using the open technique, six ports were placed, consisting of bilateral subcostal, bilateral low abdominal, subxiphoidal, and supraumbilical ports (12-mm each). A flexible electrolaparoscope (Fujinon, Tokyo, Japan) was introduced through the supraumbilical port.

The gastrectomy began with the mobilization of the greater curvature along the transverse colon, carried out with ultrasonic shears (laparoscopic coagulating shears [LCS]; Ethicon Endo-Surgery, Cincinnati, OH, 


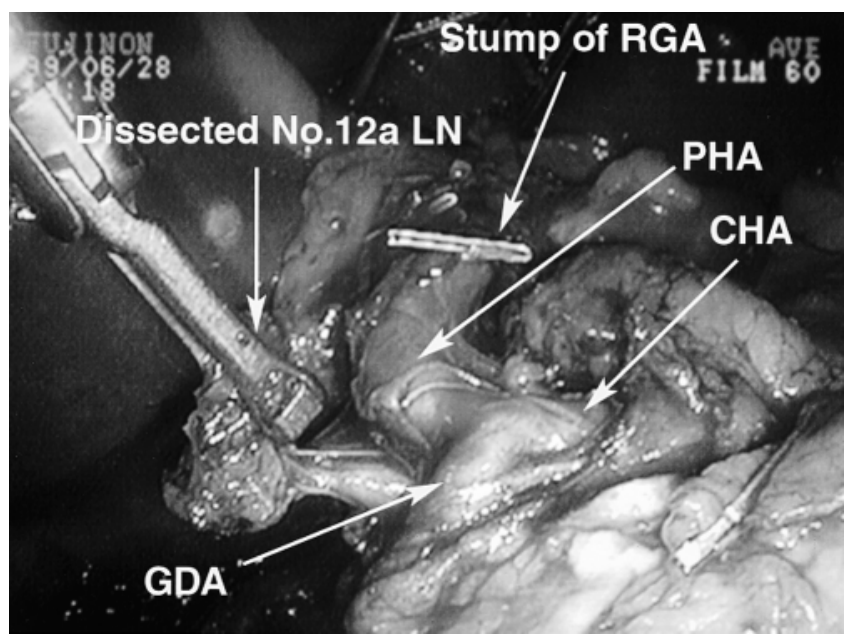

Fig. 1. Dissection of no.12a lymph node $(L N)$. $R G A$, Right gastric artery; $P H A$, proper hepatic artery; $C H A$, common hepatic artery; $G D A$, gastroduodenal artery

USA). The roots of the right gastroepiploic and gastric vessels were exposed with an ultrasonic aspirator (ultrasonic surgical unit [USU]; Olympus Optical, Tokyo, Japan), and were divided with double clips. The infra- and suprapyloric lymph nodes (LN stations nos.6 and 5, respectively) were dissected. The proper hepatic artery was exposed, and dissection of the lymph node in the hepatoduodenal ligament (along the hepatic artery) (LN station no.12a) was performed with the USU and LCS (Fig. 1).

After duodenal transection, performed with a 45-mm endoscopic linear stapler (ETS45; Ethicon EndoSurgery), the common hepatic artery was exposed, allowing for dissection of the lymph nodes along the common hepatic artery (anterosuperior group; LN station no.8a) (Fig. 2). The pancreas was isolated at the left side of the superior mesenteric vein, and transected with the ETS45 (Fig. 3). The splenic artery and vein were exposed with the USU. Subsequent to this visualization, the roots of these vessels were divided with a vascular linear stapler. The celiac and left gastric arteries were exposed, and this was followed by dissection of the lymph nodes along the celiac artery and the left gastric artery (LN stations nos.9 and 7, respectively), in a manner reported previously [11,12].

The phrenoesophageal membrane was divided and the vagal nerves were divided with the LCS. The exposed esophagus was adequately mobilized and transected with the ETS45.

The distal pancreas and spleen were dissected from the retroperitoneal space from medial to lateral with the LCS (Fig. 4). Finally, the splenophrenic ligament was divided, and an en-bloc total gastrectomy with distal pancreatosplenectomy was completed (Fig. 5). The

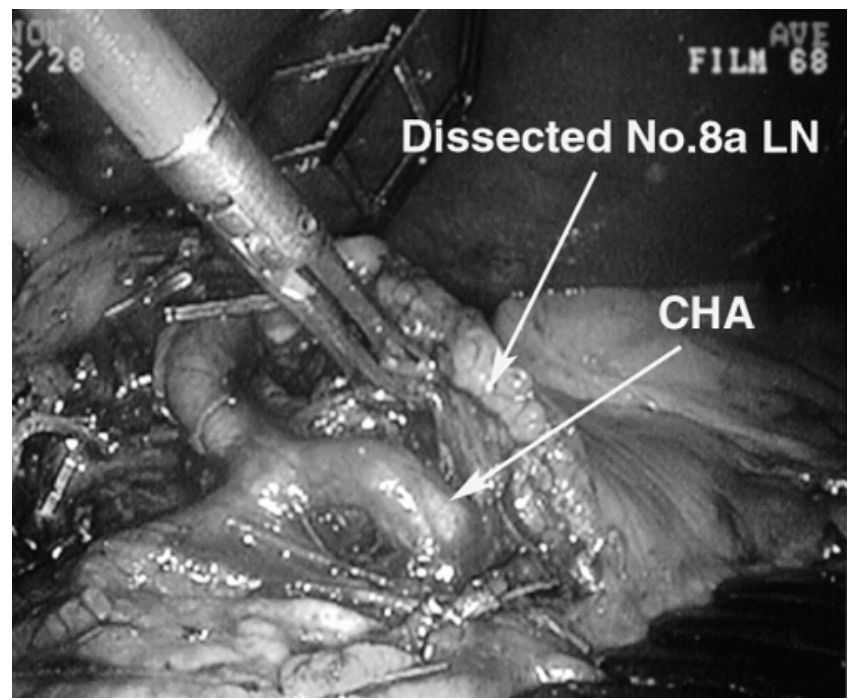

Fig. 2. Dissection of no.8a lymph node $(L N)$. $C H A$, Common hepatic artery

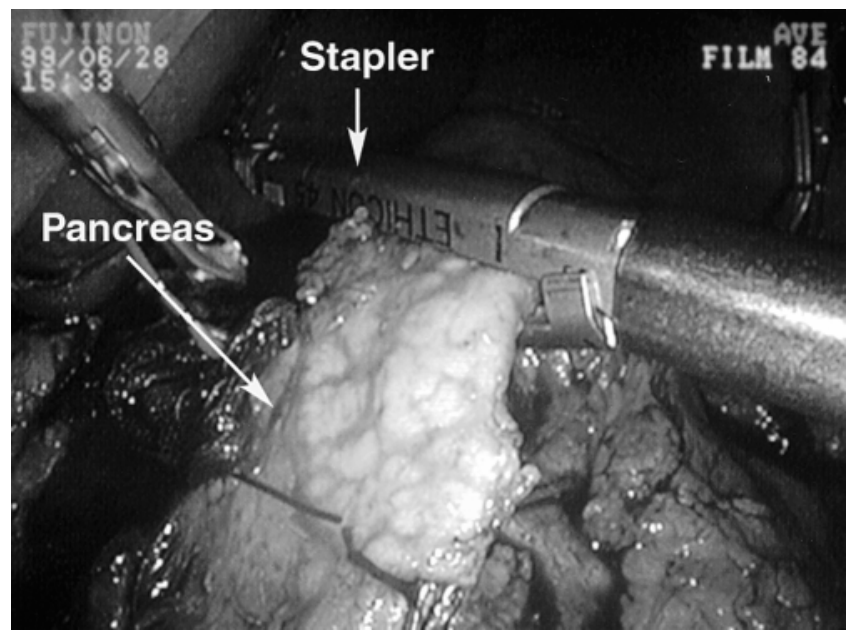

Fig. 3. Transection of the pancreas

resected organs and lymph nodes were placed in a specimen bag (Endocatch II; United States Surgical, USA), and temporarily left in the pelvic cavity (Fig. $6)$.

In the reconstruction phase of the procedure, intracorporeal anastomosis was accomplished by the Roux-en-Y method. The created jejunal loop was pulled cranially toward the esophagus via a retrocolic route. The esophago-jejunostomy was created mechanically with a functional end-to-end anastomotic technique [13]. The jejunal loop and the esophagus were excised sufficiently to allow passage of the jaws of the ETS45 (Fig. 7). The device was closed and fired, creating an anastomosis. This procedure was repeated two or three times until an adequate anastomotic lumen was 


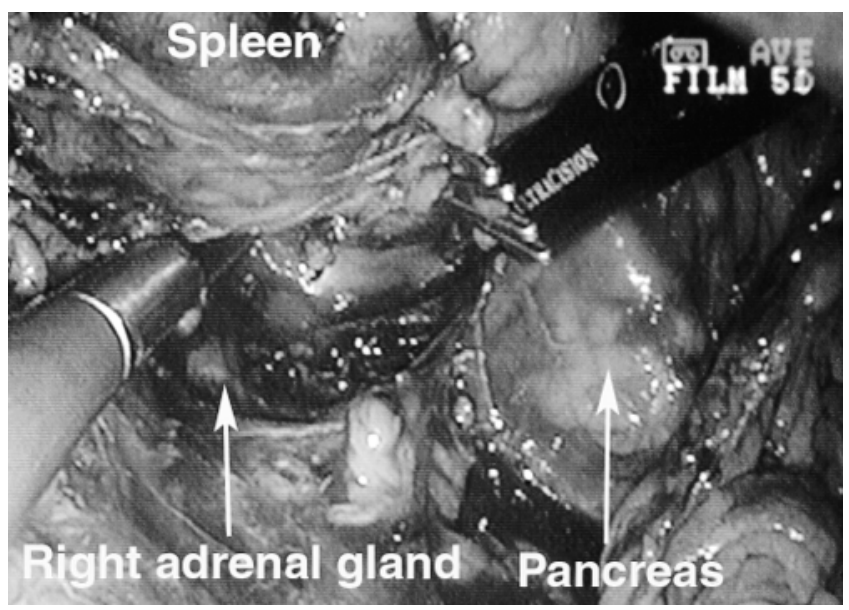

Fig. 4. Dissection of the distal pancreas and spleen from the retroperitoneal space

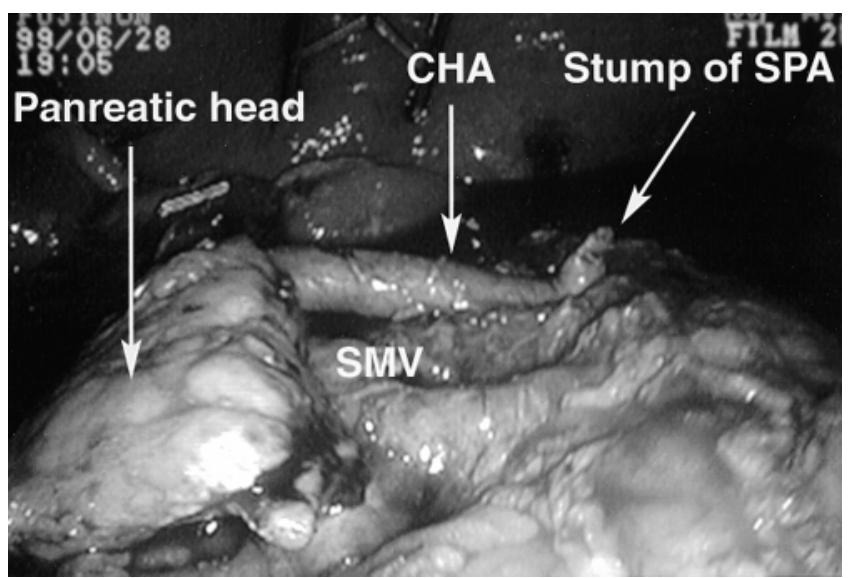

Fig. 5. Completion of en-bloc total gastrectomy with distal pancreatosplenectomy. $C H A$, Common hepatic artery; $S M V$, superior mesenteric vein; $S P A$, splenic artery

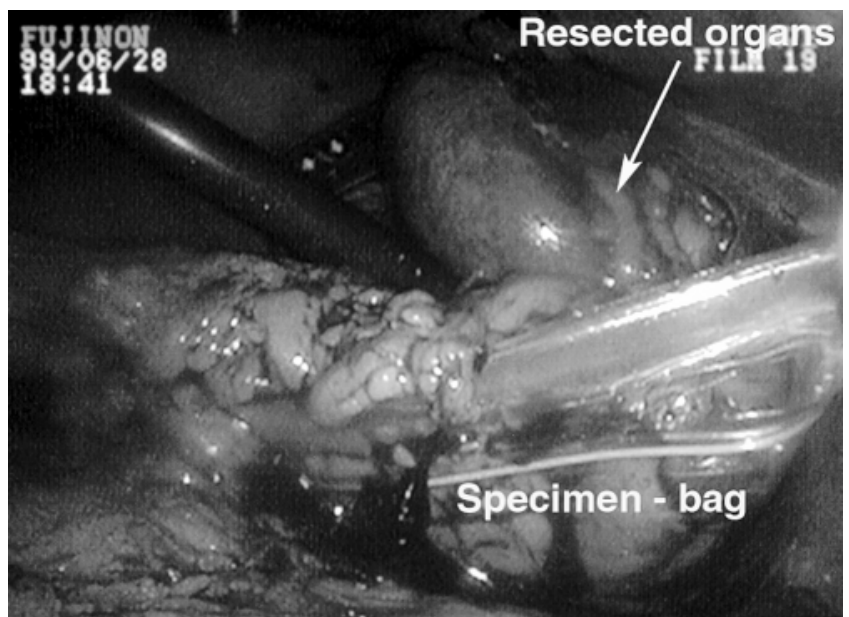

Fig. 6. The resected organs and lymph nodes were placed in a specimen bag

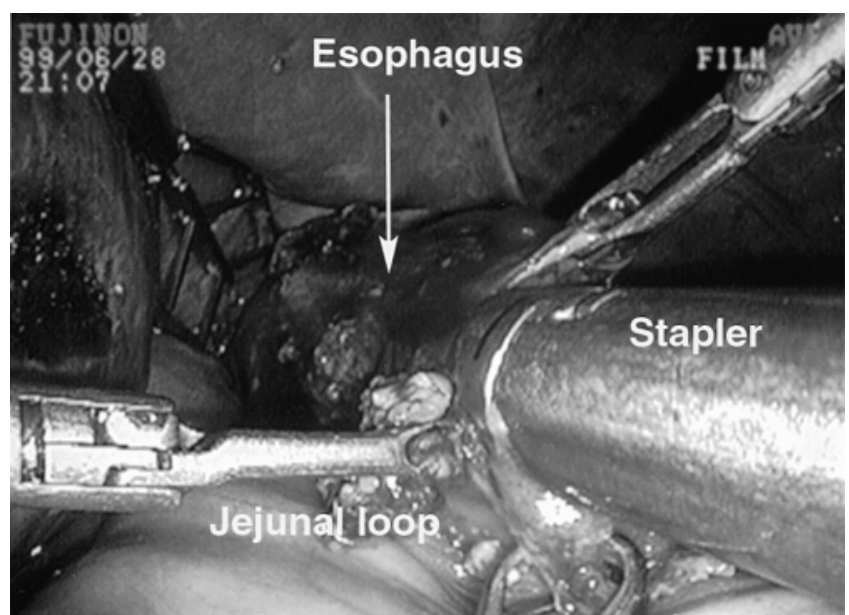

Fig. 7. An endoscopic linear stapler was inserted and positioned such that one jaw was in the jejunum and the other in the esophagus. The device was closed and fired, creating an anastomosis

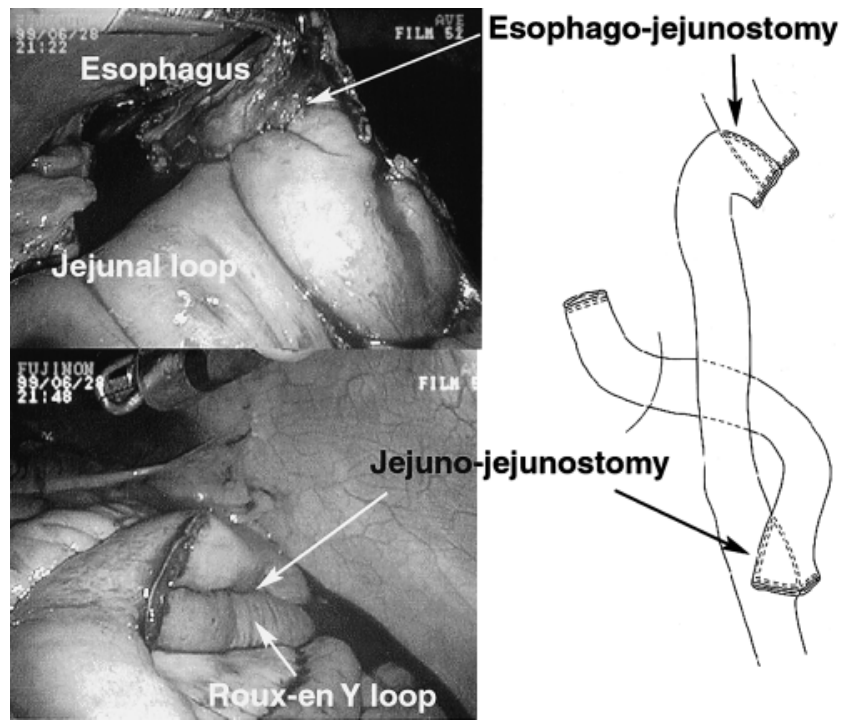

Fig. 8. Photograph and schema of the reconstruction

obtained. These firing procedures converted the two holes into one common hole, which was then closed with two or three ETS45. At this point, the esophagojejunostomy was completed (Fig. 8). The side-toside jejuno-jejunal anastomosis at the base of the Rouxen-Y loop was created $50 \mathrm{~cm}$ distal to the esophagojejunostomy mechanically, using the same stapling technique as that employed during the esophagojejunostomy (Fig. 8). The mesenteric gap was closed manually.

The left lower trocar wound was extended to $4 \mathrm{~cm}$ in length and the specimen bag was retrieved. Two drains were placed through the trocar wounds. 


\section{Results}

The two patients underwent completely laparoscopic procedures without any intraoperative complications. The operative duration was 645 and $545 \mathrm{~min}$, respectively, and amount of blood loss was 450 and $285 \mathrm{ml}$, respectively. Thus, only autologous blood transfusion was needed. The number of lymph nodes retrieved for this surgery was 63 and 58, respectively, a figure not significantly different from the number of lymph nodes retrieved with the open surgical technique. The pathology findings for the two patients' retrieved lymph nodes in each station, according to the Japanese Gastric Cancer Association "LN station number; (metastatic number/retrieved number)" were described as follows: LN station no.1, (0/6), (0/4); no.2, (0/2), (0/3); no.3, (2/ 8), (0/6); no.4sa, (0/4), (0/4); no.4sb, (0/5), (0/6); no.4d, (0/6), (0/7); no.5, (0/3), (0/1); no.6, (0/2), (0/3); no.7, (0/ 5), (0/4); no.8a, (0/5), (0/4); no.9, (0/4), (0/3); no.10, (0/ 4), (0/5); no.11p, (0/3), (0/4); no.11d, (0/4), (0/3); no.12a, $(0 / 2),(0 / 1)$, respectively. The final findings in the patients, according to the JCGC, were pT2 (mp), pN0, sH0, sP0, sCY0, fStage IB for one patient, and pT2 (mp), pN1, sH0, sP0, sCY0, fStage II for the other. In both patients, curability A, according to the JCGC, was achieved.

Both patients were pain-free and ambulatory by the second postoperative day. A fluoroscopic contrast study revealed neither leakage nor stricture of the anastomoses. There were no major postoperative complications. However, assessment of the drain effusion revealed high concentrations of amylase, consistent with pancreatic juice leakage. There was no formation of intraabdominal abscesses, nor were there any symptoms or signs of sepsis. With conservative treatment, which consisted of irrigation from the drain, the concentration of amylase had normalized by the tenth postoperative day in both patients.

The patients' postoperative hospital stays were 28 days and 31 days, respectively, although both patients could have left the hospital on postoperative day 21 . Their hospitalization was extended according to their own wishes, and not because of any medical requirements. Therefore, in a comparison of laparoscopic and open surgery, a true difference was not recognized in the duration of hospital stay.

Although in our experience the majority of patients who receive the open surgery complain of the discomfort of the operation wound after discharge, there were no such complaints in these two patients who received laparoscopic surgery. The postoperative courses of the two patients after discharge were uneventful, and they have resumed their normal daily activities without any problems.

\section{Discussion}

Although laparoscopic surgery is growing in popularity as a viable option in the management of early gastric cancer [1-6], laparoscopic gastrectomy for patients with advanced disease remains very controversial [8]. We believe this controversy is due to the following two factors: (1) although the development of minimally invasive techniques is necessary for the laparoscopic performance of the same type of operation as open D2 gastrectomy for advanced gastric cancer without any complications, this procedure is very difficult technically; (2) the laparoscopic procedure is associated with pneumoperitoneum and visceral manipulation [14].

The standard lymph node dissection for advanced gastric cancer is a $\mathrm{D} 2$ dissection, ie, the dissection of all second tier stations, and a D3 dissection is, at present, regarded as an investigational treatment [15]. Previously, we reported that laparoscopic distal partial gastrectomy with extraperigastric lymph node dissection for early gastric cancer located in the middle or lower portion of the stomach was feasible and safe [10].

Further, transection of the pancreas with a stapling device has been performed safely [16,17]. In the present series, although each patient experienced a minor pancreatic fistula, no intraabdominal abscesses were formed and the fistulas were cured with conservative therapy. Furthermore, completely intra-abdominal laparoscopic Roux-en Y reconstruction was successfully performed, using a functional end-to-end anastomotic technique. Therefore, we laparoscopically reproduced the same type of operation as an open D2 total gastrectomy, which is the standard surgery for advanced gastric cancer located in the upper portion of the stomach.

As far as port site recurrences are concerned, the literature has yet to report any such recurrences after laparoscopic radical gastrectomy. In our own 24 laparoscopic D1 or D2 gastrectomies, we have not experienced port site recurrences. However, careful visceral manipulation is very important for laparoscopic gastrectomy to be properly adapted for patients with advanced gastric malignancies. Azagra et al. [7] advised that, in order to reduce visceral manipulation and neoplastic spread, a laparoscopy-assisted approach is preferable for advanced gastric neoplasms. However, we believe that a procedure in which the extracorporeal gastric resection is performed through a mini-laparotomy cannot be done without forceful extraction of the surgical specimen. Furthermore, we assume that this forceful extraction leads to possible port site or mini-laparotomy wound recurrences, and so gastric resection has to be performed by an intra-abdominal laparoscopic approach. In this latter approach, the removal of the resected organs occurs only after their isolation in the specimen bag. 
We feel confident that laparoscopic surgery can be adapted for moderately advanced gastric cancer (up to $\mathrm{N} 1$, up to $\mathrm{T} 2$ ). However, minimally invasive surgery cannot be done in patients with severely advanced cancer (N2 or higher, T3 or higher), because of the technical difficulty involved with laparoscopic bursectomy and D3 lymphadenectomy.

Although, at present, there are problems of high cost and long operative duration associated with this procedure, completely laparoscopic total gastrectomy with distal pancreatosplenectomy and D2 lymphadenectomy is feasible and safe, and has many advantages over open surgery. Namely, it is associated with minimal postoperative pain, quicker mobilization, and better cosmesis.

\section{References}

1. Ohgami M, Otani Y, Kumai K, Kubota T, Kim YI, Kitajima M. Curative laparoscopic surgery for early gastric cancer: five years experience. World J Surg 1999;23:187-92.

2. Shiraishi N, Adachi Y, Kitano S, Bandoh T, Katsuta T, Morimoto A. Indication for and outcome of laparoscopy-assisted Billroth I gastrectomy. Br J Surg 1999;86:541-4.

3. Adachi Y, Suematsu T, Shiraishi Nm, Katsuta T, Morimoto A, Kitano S, Aazawa K. Quality of life after laparscopy-assisted Billroth I gastrectomy. Ann Surg 1999;229:49-54.

4. Kitano S, Shimoda K, Miyahara M, Shiraishi N, Bandoh T, Yoshida T, et al. Laparoscopic approaches in the management of patients with early gastric carcinomas. Surg Laparosc Endosc 1995;5:359-62.

5. Uyama I, Ogiwara H, Takahara T, Furuta T, Kikuchi K, Iida S. Laparoscopic minilaparotomy Billroth I gastrectomy with extraperigastric lymphadenectomy for early gastric cancer using an abdominal wall-lifting method. J Laparoendosc Surg 1995;5: $181-7$.

6. Kuo WH, Lee WJ, Chen CN, Yuan RH, Yu SC. Laparoscopic subtotal gastrectomy with lymphadenectomy in a patient with early gastric cancer. J Formos Med Assoc 1998;97:127-30.

7. Azagra JS, Georgen M, De Simone P, Ibanez-Aruirre J. Minimally invasive surgery for gastric cancer. Surg Endosc 1999;13: 351-7.

8. Goh PM, So JB. Role of laparoscopy in the management of stomach cancer. Semin Surg Oncol 1999;16:327-31.

9. Japanese Research Society for Gastric Cancer. Japanese classification of gastric carcinoma. 13th ed. Tokyo: Kanehara; 1999.

10. Japanese Gastric Cancer Association. Japanese classification of gastric carcinoma. 2nd English ed. Gastric Cancer 1998;1:10-24.

11. Uyama I, Sugioka A, Komori Y, Fujita J, Hasumi A. Laparoscopic distal gastrectomy with intracorporeal D2 lymph node dissection for early gastric cancer. In: Montori A (ed). Proceedings of the Sixth World Congress of Endoscopic Surgery, held in Rome, Italy, June 3-6, 1998. Bologna: Monduzzi Editore; 1998. p. 633-6.

12. Uyama I, Sugioka A, Fujita J, Komori Y, Matsui H, Soga R, et al. Completely laparoscopic extraperigastric lymph nodes dissection for gastric malignancies located in the middle or lower third of the stomach. Gastric Cancer 1999;2:186-90.

13. Steichen FM. The use of staplers in anatomical side-to-side and functional end-to-end enteroanastomoses. Surgery 1968;64:94853.

14. Whelan RL, Lee SW. Review of investigations regarding the etiology of port site tumor recurrence. J Laparoendosc Adv Surg Tech A 1999;9:1-16.

15. Aiko T, Sasako M. The new Japanese classification of gastric carcinoma: points to be revised. Gastric Cancer 1998;1:2530.

16. Pachter HL, Pennington R, Chassin J, Spencer FC. Simplified distal pancreatectomy with the auto suture stapler: preliminary clinical observations. Surgery 1979;85:166-70.

17. Lukish JR, Rothstein JH, Petruzziello M, Kiteley R, Denobile J, Soballe P. Spleen-preserving pancreatectomy for cystic pancreatic neoplasms. Am Surg 1999;65:596-9. 